\title{
La participación ciudadana y su educación: nuevos retos en la era digital
}

Citizen participation and education: new challenges in the digital age

Rocío González-Andrío Jiménez, Irene Palomero Ilardia (*)

\section{Resumen}

Se presenta una aproximación al concepto de participación ciudadana y su educación. En una sociedad c ambiante, g lobal, di gital en la que la democratización de l conocimiento ya es un a realidad, s e ac recientan I os r etos educ ativos en un contexto d e om nipresencia de I o di gital entre l os jóvenes. Se incide en la importancia d e i ncorporar en la asignatura de $\mathrm{C}$ iencias Sociales en Primaria y de Geografía e Historia en Secundaria una educación ciudadana para saber participar de manera offline y online.

Palabras Clave: Participación c iudadana, Educación c ívica, C iudadanía digital, D emocracia, Prácticas educativas.

Abstract

The main obj ective of $\mathrm{t}$ his ar ticle i s t o ex plain the $\mathrm{c}$ oncept of $\mathrm{c}$ itizen par ticipation a nd its education. I n o ur $\mathrm{c}$ hanging, global, and $d$ igital s ociety $\mathrm{i}$ which $\mathrm{t}$ he $\mathrm{d}$ emocratization of knowledge is a r eality, educational challenges are increasing in the context of om nipresence digital means among young people. This article em phasizes the importance to include, in the subject of Social Sciences, in Primary, and in the subject of Geography and History, in Secondary levels, a citizenship education to teach how to participate offline and online.

Key words: Citizen participation, C ivic ed ucation, Digital c itizenship, D emocracy, E ducational practices

\section{Introducción}

El artículo tiene como objetivo indagar sobre el concepto de pa rticipación ciudadana en plena era digital, en la que se acr ecientan los retos educativos de una so ciedad cambiante, co n m ás oportunidades de par ticipación so cial onl ine y en l a q ue la libertad ${ }^{1}$ de expresión es uno de los valores más apreciados (Rowbottom, 2006).

\footnotetext{
${ }^{1}$ Cuando hablamos de libertad lo entendemos como toda ausencia de imposición o coacción externa o de intimidación.

1.Universidad Rey Juan Carlos, Grupo de investigación emergente Laboratorio de Innovación y didáctica aplicada (GI-LIDA). Rocío González - Andrío Jiménez ORCID 0000-0001-9548-8210, rocio.gonzalezandrio@urjc.es, Irene Palomero Ilardia ORCID 0000-0001-7197-7431, Irene.palomero@urjc.es
}

González-Andrío, J, R.; Palomero llardia, I. (2021). La participación ciudadana y su educación: nuevos retos en la era digital, Clio. History and History Teaching, 47, 85-100. https://doi.org/10.26754/ojs clio/clio.2021475842 Recibido 24/7/2021, Aceptado $31 / 10 / 2021$ 
La participación y l a ci udadanía so $\mathrm{n}$ I os dos temas ce ntrales de l a democracia. Participación, porque es el pueblo y en concreto son sus ciudadanos y ciudadanas los que, de un m odo I ibre y act ual, pueden t omar par te en I as decisiones que l es incumben a nivel social, político, económico, cultural, o en relación a temas que hoy nos inquietan, como es el futuro de la educación o la salud pública. Palabras, por tanto, de difícil separación en la teoría, aunque en la práctica, a lo largo de la historia, desde la an tigua Grecia hasta nues tros días, es una cl ase pr ivilegiada la q ue ha ejercido o ejerce el derecho de participación (Heater, 2007). El perpetuo conflicto entre libertad y compromiso cívico ha dado lugar a revoluciones sociales desde el siglo XVIII hasta nuestros días (Costa, 2006) y son los cambios sociales, así como los diferentes modos de pensar, lo que ha impulsado las constantes reivindicaciones por la igualdad y la no exclusión de persona alguna en los procesos de participación política, social y ciudadana ${ }^{2}$.

Esta búsqueda de la igualdad social comienza por lo más material y se va ampliando al resto de las necesidades básicas de la vida de las personas (Buitrago et al. , 2007), hasta llegar a I a I ibertad de ex presión y participación, en la búsq ueda de un bi en individual y común. De este modo, en pleno siglo XXI con el uso de Internet y las redes sociales, la forma de gobernar en I os países democráticos está cambiando. Es decir, se está dando una mayor hor izontalidad g ubernamental, en la que juegan un pape I clave no solo los empresarios y empresarias, las organizaciones públicas $u$ organizaciones no g ubernamentales, si no t ambién la ci udadanía, que puede ahor a opinar, escribir y aportar soluciones desde su casa, con un or denador o di spositivo móvil y con una conexión a Internet (González et al., 2016). De acuerdo con La Porte (2019), el fomento de la par ticipación y co laboración de I os ciudadanos con I as organizaciones favorece la escu cha, la reflexión y el debate de I as propuestas. A sí, cuando I os gobiernos permiten y facilitan a I os ciudadanos desarrollar y ej ercer sus capacidades, habilidades personales y co mpetencias cívicas ${ }^{3}$, es entonces cuando

\footnotetext{
2 Tenemos como ejemplo el levantamiento del pueblo cubano que se inició en julio del 2021 U. C. 7/21 https://elpais.com/opinion/2021-07-21/una-nueva-cuba-quiere-vivir.html

3 La palabra civismo se puede definir como un conjunto de capacidades, de habilidades, actitudes y competencias que hacen de las personas "aptas" para formar parte de comunidades políticas en las que se integran. El comportamiento cívico de las personas ha sido estudiado desde una doble vertiente: teórica (Cómo debería ser) y práctica (Cómo es en la realidad) (GarcíaGalera, del-Hoyo-Hurtado, \& Fernández-Muñoz, 2014, Lizcano Fernández, 2012).
} 
cobra se ntido I a i dea de I ibertad, que i mplica t oda par ticipación so cial y c ívica ${ }^{4}$. Bennett ( 2011) distingue dos clases o maneras de ejercer y entender la ciudadanía. A la primera la denomina Dutiful Citizenship (DC), una ciudadanía que se comporta de manera responsable y animada por la idea del deber. La segunda, la llama Actualizing Citizenship (AC) y la identifica con una ciudadanía jóven que "actualiza" sus intereses ciudadanos at ravés de $r$ edes en I nternet y cu yos estilos de apr endizaje se caracterizan por la interactividad.

Gran número de autores reflejan cómo el desarrollo de la comunicación digital continúa abriendo nuevas e insospechadas perspectivas en el campo de la educación, la política, la economía, la vida social y concretamente en el de la participación ciudadana online y offline (Bakker y de Vreese, 2011). Así, uno de los retos educativos sigue si endo ens eñar a pensa $r$ de manera reflexiva y crítica en I a er a del conocimiento, en el que la elaboración de los contenidos en línea, es producto de la contribución de los ciudadanos.Tengamos en cuenta que a partir del año 2000 es cuando se generaliza el uso de las redes sociales y se empieza a hablar en en ámbito académico de retos educativos vinculados al uso de las nuevas tecnologías (Prados et al., 2015; Touriñán López, 2001).

\section{Dificultades para definir y explicar que es la participación ciudadana}

Ambos conceptos, participación y ciudadanía, tienen una l arga historia. Sin embargo, la def inición y de limitación co nceptual de par ticipación ci udadana es una t area compleja. En primer lugar, no existe acuerdo en lo que es y lo que no es la acción de participar como ciudadano o ci udadana. En segundo lugar, el problema reside en la gran $\mathrm{v}$ ariedad de e xperiencias osi tuaciones participativas, in cluidas las ya mencionadas, llamadas virtuales u online. Por úl timo, es la relación i mprecisa ent re Estado y sociedad civil, así como la ambigüedad del significado de ambas palabras, lo que di ficulta da $r$ una d efinición ce rrada de di chos conceptos (Baño, 1998; $P$ arés Franzi, 2009). Aun a sí, I as definiciones encontradas de par ticipación ci udadana apuntan cu atro ideas que nos pueden a yudar a enm arcar el t ema. L a primera, el significado de la palabra participación (de raíz latina, particeps, "entrar a la parte"), hace referencia a tomar parte en y con otros en asuntos de bien común, que incluye

\footnotetext{
${ }^{4}$ Sobre el gobierno abierto y la transparencia en la información digital, ver Curto-Rodríguez, R. (2020). Gobiernos autonómicos españoles y transparencia activa de sus repositorios digitales. Una revisión taxonómica. Política y Sociedad, 57(2), 543.
} 
los intereses tanto personales como colectivos; de tal modo que, en toda participación se est ablecen ci ertas formas de co laboración. La se gunda, I a par ticipación i mplica interés y voluntad de querer colaborar con, por lo que la noción tiene, en principio, una aceptación y connotación positiva. La t ercera, el principio de par ticipación, es el eje sobre el que gira y da vida al compromiso social, por lo que (García-Galera et al., 2014) se excluye de la idea de participación toda acción que conlleve cualquier tipo de manipulación social individual o colectiva. Finalmente, lo central del tema es quiénes y cómo se participa, teniendo en cu enta que en e I célebre artículo "A ladder of citizen participation", se clasifican los niveles de participación y se vincula el concepto de participación ciudadana con el poder que tiene la ciudadanía en sus manos (Arnstein, 1969).

Si bi en no es lo $\mathrm{m}$ ismo par ticipación pol ítica que par ticipación ci udadana, so $\mathrm{n}$ dos expresiones que están i ntrínsicamente uni das. $P$ ara $P$ arés Franzi ( 2009), I a participación ciudadana son todas las acciones de tipo político y social en las que la ciudadanía, de un modo claro, busca influir. Otros autores subrayan la m isma idea, con el verbo incidir en todo proceso de participación (Cohen y Ghosh, 2019; Heater, 2007; Marshall \& Bottomore, 1992; Ichilov, 2013), pero no hay una frase o una palabra exclusiva que identifique o defina a la participación ciudadana.

En cuanto al concepto de ciudadanía, Delgado-Algarra (2015) lo explica en función de una serie de variables, incluída la de la participación. Estas otras variables son las de los derechos y responsabilidades, la importancia individual y comunitaria, los enfoques locales, nacionales e internacionales, la crítica o la ideología (Delgado-Algarra et al, 2019a; Delgado-Algarra et al., 2019b; Delgado-Algarra et al. 2020a). Pero sigue siendo un t érmino en co nstrucción y em inentemente su bjetivo ( Delgado-Algarra, et a l., 2020b). S in em bargo, podemos resumir el doble r ol que juega la ciudadanía en: a) Llevar I as riendas del g obierno, dem andando una respuesta co ncreta a I as necesidades y requerimientos de la ciudadanía y b) Cumplir las "reglas" ciudadanas bajo las que viven. Este doble papel nos recuerda por un I ado, có mo e n la antigua Grecia , a los que er an considerados ciudadanos, unas veces les tocaba mandar y otras ser mandados, como tareas alternantes, en una co munidad co ncreta (Heater, 2007a) y por otro a los dos tipos de ciudadanos clasificados por Bennett, 
anteriormente m encionados, I os llamados Actualizing Citizenship (AC) y I os Dutiful Citizenship (DC).

\section{Participación y compromiso ciudadanos}

El poder ciudadano, al que ya hemos hecho referencia, tiene de igual manera una doble cara: la de los derechos y la de las obligaciones. Si retrocedemos en el tiempo, en el año 1965, Milbrath publicó un libro titulado Political Participation: How and Why do People Get Involved in Politics, en el que repasa la literatura existente de esos años sobre el tema de la participación ciudadana en la política. Concluye que la mayor parte de los americanos en edad adulta piensan que, para que la democracia prospere en un país, es esencial que los ciudadanos y ciudadanas tengan cierto interés en los asuntos políticos, lo que supone cierto compromiso personal. Deben, además, saber informarse y $f$ ormarse para I legar a se $r$ ci udadanos activos en cuanto a su participación pol ítica (Milbrath, 1965). Han pasa do ca si cincuenta y se is años desde esta afirmación y hoy podemos decir que I a ex presión par ticipación pol ítica y ciudadana, en relación con el compromiso social y la educa ción cívica, los modos y medios de ejercer ese poder ciudadano, sigue siendo parte del debate actual (Cohen y Ghosh, 2019).

Llegados a est e pun to, nos podemos preguntar qué es el co mpromiso so cial. Comencemos por recordar que cuando hacemos referencia a la participación política de los ciudadanos, pensamos en los motivos que animan a la ciudadanía a involucrarse o comprometerse en los asuntos públicos. Las motivaciones son variadas: inquietudes de tipo pol ítico, eco nómico o so cial, co mo es la pr eocupación por los niveles de desempleo actual; temas relacionados con lo educativo; asuntos de justicia social, medio ambientales, o cuestiones con un fondo ético que alcanza la conciencia de las personas, como son la violencia de género o la pobreza material e intelectual en la que vive una buena parte de la sociedad española. $S$ abemos que cuando a los ciudadanos les preocupa un asunto que concierne de un modo directo a sus vidas, es cuando se ha dado un aumento de la participación en forma de movimientos sociales online o offline (Calzada, 2017; Sádaba, 2012).

Con t odo, la pal abra co mpromiso pa rece que no está muy de moda hoy en dí a, al entenderse en algunos casos y entre determinados segmentos de población más 
joven, como una limitación de la libertad personal. La de finición de co mpromiso de la Real Academia de la Lengua Española, como obligación contraída o palabra dada, no incluye dicha limitación en ninguna de sus acepciones. El compromiso siempre debe ser libre. Esta idea, aun que básica, se debería tener en cu enta a I a hor a de educa $r$ para una participación ciudadana. Si el compromiso es explicado desde un punto de vista positivo, como modo de co laboración so cial en el que la persona se realiza al desarrollar todas sus capacidades al servicio de la comunidad y al mismo tiempo pone en ejercicio el poder que se le otorga como ciudadana o ciudadano, el problema de la desidia o apat ía so cial podr ía co menzar a so lucionarse ( Hernández M erayo, 2011 ; Jover, 2016; Vizcaíno-Laorga et al., 2019).

Respondemos al a p regunta i nicial de est e apar tado. ¿Qué es el co mpromiso ciudadano? De manera resumida, podemos decir que es la oportunidad que tiene toda persona para desarrollar todas sus capacidades, de un modo libre y autónomo, para ponerlas al servicio de la sociedad.

\section{Tipos de participación ciudadana}

Como se ha señalado anteriormente, no se puede decir que exista un consenso absoluto sobre lo que es la participación política o ciudadana. Y es que, como sucede con ot ros muchos conceptos, su uso y abuso pueden dese mbocar en un vacío o confuso si gnificado et imológico. $S$ in e mbargo, so n co nceptos, en cierto modo, delimitables, si encontramos por ejemplo, una clasificación de acciones concretas consideradas como modos de participación pol ítica y ci udadana (Sabucedo, 1988). Por eso, la pregunta clave que se hacía Milbrath (1965) es cómo la gente se involucra.

En la clasificación de los tipos de ci udadanos de Westheimer y Kahne (2004), I os ciudadanos personalmente responsables son Ias personas que, por ejemplo, pagan los impuestos; ciudadanos participativos en su comunidad son los que ayudan a organizar y trabajan cooperativamente con los demás; por último, los ciudadanos que están orientados hacia la justicia social son los que se preguntan por las raíces de los problemas para solucionarlos. Ésta es una de las tipologías que apoyan la idea de 
que, para que una acci ón concreta sea incluida en di cha participación pol ítica, debe tener al m enos como fin querer incidir en I as decisiones últimas que van a $t$ omar nuestros gobernantes.

La era digital ha abierto nuevos modos de participación social y ciudadana dando lugar a lo que hoy Ilamamos ciudadanía digital (Prensky, 2001; Ribble et al, 2004; Robles Morales, 2009). La cuestión clave es entender y conocer cómo se está dando este tipo de par ticipación di gital y los retos que nos plantea, en tre los que se encu entra aunque se est á avanzando en est e ca mpo-, I a falta de no rmativa y de leyes que regulen todo lo que se puede o se debe "hacer" en el espacio virtual. En una de las definiciones de ciudadanía de la Real Academia Española, la persona es considerada como miembro activo de un Estado, titular de derechos políticos y sometido a sus leyes. Esta definición nos puede servir para cerrar este apartado y preguntarnos ¿Están pr eparados los est udiantes y sa ben có mo, dónde y el por qué de I a participación offline y online? ¿Cuáles son las capacidades y habilidades que deben adquirir para enfrentarse hoy a los nuevos modos de participación ciudadana digital?

\section{Razones para educar en participación ciudadana}

Hemos explicado cómo la participación ciudadana es algo necesario para que el sistema democrático de un país funcione correctamente, sin olvidar que toda forma de participación co nlleva desafíos aun en g obiernos democráticos (Jover, 2016) . Asimismo, para que pueda darse una participación ciudadana, se requieren las estructuras gubernamentales y adm inistrativas necesarias, como los canales de participación para poder ejercer el derecho de manera responsable ${ }^{5}$. Asimismo, cuando hablamos de participación ciudadana en Ios asuntos públicos, Ios conceptos de autonomía, libertad e igualdad de clases son lo que distingue a la ciudadanía de otras formas de identidad sociopolítica (Heater, 2007b).

\footnotetext{
${ }^{5}$ En el portal de Internet de transparencia de la Administración General del Estado, en la pestaña de participación pública se pueden encontrar los artículos de las leyes que explican los espacios de participación pública. Esta participación está recogida en el artículo 133 de la Ley 39/2015, de 1 de octubre, del Procedimiento Administrativo Común de las Administraciones Públicas y en el artículo 26 de la Ley 50/1997, de 27 de noviembre, del Gobierno, así como en la Orden PRE/1590/2016, de 3 de octubre, por la que se publica el Acuerdo del Consejo de Ministros de 30 de septiembre de 2016, por el que se dictan instrucciones para habilitar la participación pública en el proceso de elaboración normativa a través de los portales web de los departamentos ministeriales. https://transparencia.gob.es/transparencia/transparencia Home/index/ParticipacionCiudadana/Participacion ProvectosNormativos.html U. C. 25/6/2021
} 
Toda persona tiene derecho a participar en el gobierno de su país, directamente o por medio de representantes libremente escogidos, como dicta el artículo 21 de la Declaración U niversal de I os Derechos del H ombre, que i ntroduce en I a hi storia universal el concepto de participación ciudadana. En las consideraciones del Preámbulo de la Declaración, la libertad, la justicia y la paz son reconocidas en base a la dignidad de la persona y de los derechos iguales e inalienables de todos los hombres y mujeres. Es decir, que todos los miembros de la familia humana a la que hace $r$ eferencia I a D eclaración t ienen el der echo de par ticipación y que, por se $r$ considerados éstos fundamentales, pode mos afirmar que so $n$ ne cesarios para el desarrollo de la persona en su totalidad. Igualmente, en los principios constitucionales recogidos en el Título Preliminar de nuestra norma fundamental (art. 9.2 de la Constitución Española ${ }^{6}$ ), se concreta como un derecho fundamental el de participación en I os asuntos públicos (art. 23.1 de I a $C$ onstitución $E$ spañola). P or t anto, I a participación ci udadana es uno de I os principios fundamentales del o rden jurídicopolítico que configuran el Estado.

Estas ideas tienen su ficiente peso, si endo un argumento $v$ álido par a si tuar a la educación en participación ciudadana en el lugar que le corresponde en la enseñanza, llena de opor tunidades par a que el al umnado apr enda a pensa $r$ y a co municar, entienda las causas que han llevado a participar a los ciudadanos y ciudadanas a lo largo de I a historia de u na manera directa o indirecta en I a búsqueda de un ca mbio social, económico, legal o ético y las consecuencias que dichas actuaciones como son los giros en el $r$ umbo de I a hi storia de I a hum anidad. E jemplos actuales son I os movimientos sociales por el cambio climático en todas partes del mundo en el año 2019, los Ilamados chalecos amarillos en Francia 2018 o el movimiento $15 \mathrm{M}$ de I os indignados en Madrid en el año 2011, convocados todos ellos desde las redes sociales por diversos agentes sociales.

\section{Educación para la participación ciudadana en el aula de Primaria y Secundaria}

Los ciudadanos y ci udadanas virtuales son todos aquellos internautas que, desde cualquier di spositivo co $\mathrm{n}$ acce so a Internet, pu eden par ticipar co $\mathrm{n}$ texto, mensajes, imágenes, videos o co mentarios en línea (González-Andrío Jiménez, et al., 2020) y

\footnotetext{
6 "Corresponde a los poderes públicos [...] facilitar la participación de todos los ciudadanos en la vida política, económica, cultural y social".
} 
aunque es la i nteracción un a de las palabras claves que co ncreta el ac tuar del ciudadano di gital en una cibercomunidad, es la ca pacidad de u tilizar Internet pa ra participar como ciudadanos democráticos y la posibilidad de influencia que nos ofrece Internet para conseguir una igualdad de derechos, la que mejor la define ${ }^{7}$ sin olvidar que el desarrollo de competencias específicas, como es la comunicativa digital, es algo esencial en todo proceso participativo como ciudadanos en línea.

Son di versas las lagunas que enco ntramos en el alumnado cu ando I legan a I a Universidad. Una de ellas es la gran dificultad que tienen, en ocasiones, para expresarse co rrectamente, participar, comunicar, ar gumentar o ex plicar co nceptos básicos de manera oral y por escrito. Si estas habilidades, entre otras, son necesarias para desenvolverse adecuadamente y ser escuchados en cualquier proceso participativo, uno de los retos educativos es enseñar a comunicar de manera offline y online para saber participar, lo que conlleva por parte del profesorado: reconocer la necesidad de un cambio profundo en el proceso de enseñanza-aprendizaje y que éste sea $\mathrm{m}$ ás participativo y activo para co nocer có mo pi ensan l os estudiantes, $\mathrm{m}$ ás vivencial, experimental y digital; saber integrar los nuevos modelos o paradigmas del aprendizaje basados en los intereses de las nuevas generaciones que "viven" en un contexto di gital; implementar, junto a I as nuevas metodologías del apr endizaje, un modelo de escuela y de programación que ofrezca respuestas a las necesidades de un mundo global e interconectado, en el que la democratización del conocimiento es consecuencia de los nuevos modos de comunicación social.

Pero demos respuesta a una pregunta para concretar las ideas anteriores: ¿Cómo trabajar, por ejemplo, la participación ciudadana digital en el aula de Ciencias Sociales, para la etapa de Educación Primaria, y de Geografía e Historia, en la etapa de Educación Secundaria? Entendemos que los programas educativos son demasiado extensos y el t iempo escaso, po $\mathrm{rl}$ o que, $\mathrm{n}$ o poca $\mathrm{s}$ veces se de scarta de l a programación la parte que corresponde a una educación en participación ciudadana. Sin embargo, siguen si endo actuales las pal abras de Pagés (2002): es tiempo de repensar y transformar l a formación del profesorado que ense ña hi storia y ci encias sociales, para conseguir que el alumno construya sus propios conocimientos, aprenda

\footnotetext{
${ }^{7}$ No olvidemos el tema de la brecha digital que ha quedado de manifiesto durante la Pandemia COVID 19.
} 
a pensar, se ubique en el mundo y esté preparado para intervenir en é I de m anera democrática, con la ayuda insustituible de sus maestros y profesores.

Para responder a I a pr egunta de có mo t rasladar al aul a I a ense ñanza de I a participación ciudadana en primer lugar, tenemos que justificar desde el punto de vista didáctico, la idoneidad de implementar esta temática y analizar el currículo educativo en $\mathrm{v}$ igor pa ra ca da un a de $\mathrm{I}$ as etapas mencionadas, que $\mathrm{t}$ iene $\mathrm{v}$ inculación co $\mathrm{n} \mathrm{I} a$ educación en participación ciudadana y habilidades digitales.

En el currículo oficial de Primaria vigente en la actualidad, encontramos un bloque temático pr opio en el que i nsertar el t ema: "Vivir en S ociedad". Este bloque de contenidos abarca aspectos como la Educación vial y las normas de circulación, temas de $\mathrm{G}$ eografía $\mathrm{E}$ conómica ( sectores de pr oducción) y H umana (movimientos migratorios) y la Educación financiera, hasta la organización social, política y territorial del E stado esp añol, I os der echos y deber es de I os ciudadanos, I a C onstitución de 1978 o la Monarquía Parlamentaria. Si examinamos los criterios de evaluación y los estándares de aprendizaje evaluables de estos últimos temas, se observa que lo que se debe lograr es que los alumnos conozcan, identifiquen y comprendan la importancia de las principales instituciones del Estado, la división de poderes, explicando además la organización territorial de España. Asimismo, se pretende que identifiquen, respeten y valoren los principios democráticos más importantes establecidos en la Constitución, siendo conscientes de la importancia que ésta tiene para el funcionamiento del Estado español $^{8}$. E n el bl oque dedicado a I a $\mathrm{H}$ istoria de est a misma et apa e ducativa, el período hi stórico co $\mathrm{n}$ el q ue se co rrespondería est a t emática apar ece $\mathrm{t}$ ambién recogido, si bien lo que se pretende, al menos a nivel estatal y sin perjuicio de lo que pueda especificar la normativa propia de cada autonomía, es que los discentes sepan "identificar y localizar en el tiempo y en el espacio los procesos y acontecimientos históricos más relevantes de la historia de España para adquirir una perspectiva global de su evolución" (RD 126/2014), concretándose, para el período objeto de análisis, en que el al umno o al umna que termina la et apa Primaria, se a capaz de describir "los rasgos característicos de I a so ciedad esp añola act ual, y valora su ca rácter democrático y plural [...]" (RD 126/2014). Por lo tanto, podemos afirmar que el bloque de "Vivir en Sociedad" sería el más apropiado, aunque no el único, para introducir el

\footnotetext{
${ }^{8}$ Real Decreto 126/2014, de 28 de febrero, por el que se establece el currículo básico de la Educación Primaria.
} 
trabajo co n co nceptos básicos como de mocracia, $C$ onstitución, ci udadanía, participación ciudadana, etc. Muchas de las actividades que se realicen fuera y dentro del a ula para explicar estos contenidos pueden ser trabajadas con una metodología participativa en I a que los medios digitales, como pueden se $r$ las redes sociales, ya que es la tercera competencia, la digital citada en el Real Decreto, después de la lingüística y la matemática. Los trabajos pueden incluir actividades en I as que los "detectives historiadores", a través de procesos de análisis y síntesis de contenidos e información en Internet, se presenten no solo en papel sino también en soporte digital, utilizando programas educativos digitales como $m$ aterial de apoy o pa ra descu brir y realizar proyectos, creando y comparando documentos digitales (texto, imagen, video y sonido). Se trata de enseñar a buscar, seleccionar y analizar con sentido crítico la información $r$ elevante, ut ilizando I a her ramienta t ecnológica a decuada y compartiéndolo con sus compañeras y compañeros de clase. ${ }^{9}$

En relación con la etapa de Secundaria, del análisis del currículo ${ }^{10}$ podemos observar que ya no aparece el bloque de "Vivir en Sociedad" y el trabajo se centra en aspectos de I a $\mathrm{H}$ istoria y I a $\mathrm{G}$ eografía. $\mathrm{C}$ ierto es que e $\mathrm{n}$ est a e tapa edu cativa se am plía y profundiza más en los temas de estas dos disciplinas a lo largo de los cuatro cursos, pero podem os encontrar una m ención a det erminados contenidos vinculados con la participación ci udadana y el co ncepto de ci udadanía dent ro del bl oque oct avo ' $E$ l mundo reciente: siglos $X X$ y $X X I$ ' y, en parte, el bloque décimo 'Relación entre pasado, presente y futuro a través de la Historia y la Geografía'. Sin embargo, también se debe puntualizar que el enfoque que se da es más global y eurocéntrico.

Por otro lado, en esta etapa educativa, concretamente en el tercer curso, se trabaja el período histórico de la transición política en España y, específicamente, el paso de la Dictadura a la Democracia y la Constitución de 1978. En este tema, enmarcado dentro del bloque de Historia, consideramos algo crucial que, además de analizar y comprender los hitos que marcaron el proceso histórico de l a transición, se puedan reforzar en el aula los conceptos de participación ciudadana, democracia participativa, Monarquía Parlamentaria, Estado Autonómico o separación de poderes. No se trata de

\footnotetext{
9 Herramienta digitales para la enseñanza de la Historia y Geografía: Google maps, Radio Garden o Google Earth, https://www.classtools.net/FB/home-page 0 entre otras muchas https://artsandculture.google.com/story/WwURFnRfOCnVEQ

10 Real Decreto 1105/2014, de 26 de diciembre, por el que se establece el currículo básico de la Educación Secundaria Obligatoria y del Bachillerato.Vigente hasta el curso 2021/22.
} 
olvidar la cronología o el est udio y las consecuencias que tuvieron a contecimientos históricos como I a m uerte de F ranco, I a firma de I os Pactos de I a Moncloa, I os atentados terroristas durante la dictadura, la transición y parte de la democracia, o el fallido golpe de estado de 1981, sino de incorporar en todos ellos la reflexión y el pensamiento crítico, por parte de los alumnos, sobre lo que supusieron los conceptos antes mencionados en el avance de la ciudadanía española hacia un nuevo sistema organizativo pol ítico, en el que se tiene la opción de par ticipar de una manera libre, activa y co $\mathrm{n} g$ arantías jurídicas. D el anál isis detallado del cu rrículo of icial vigente, podemos afirmar que la participación ciudadana, la ciudadanía, encajan en los diseños docentes de las etapas de $\mathrm{E}$ ducación P rimaria y E ducación S ecundaria O bligatoria, dentro de asignaturas troncales en ambos casos, por lo que pueden implementarse sin problema en las aulas.

Por último, entendemos que el mejor modo de incorporar una educación para la participación ciudadana es a través de actividades que impliquen de manera directa al alumnado dent ro y fuera del aul a, co mo s on I as realizadas desde di ferentes administraciones públicas, a nivel nacional, europeo y mundial, en la que se plantean diferentes propuestas, proyectos, encuestas de opinión a los ciudadanos y ciudadanas bajo un concepto de colaboración y espacios para fomentar la participación social.

\section{Conclusiones}

La participación ciudadana es necesaria para el buen funcionamiento de la democracia y la e-democracia. Las posibilidades de participación social parece que se han ampliado con el creciente aumento del uso de los medios digitales y las modalidades de acce so a I a información di gital en I os portales gubernamentales. Las dificultades para incorporar en el a ula la ense ñanza de un a ciudadanía participativa y di gital no son pocas. El reto parece que comienza por superar dichos inconvenientes adaptando los recursos de los que disponemos para incorporar en asignaturas como las Ciencias Sociales la enseñanza y práctica de una participación online y offline de una $m$ anera cívica y co mprometida. Hemos hecho hi ncapié en I a nece sidad de desa rrollar una serie de habilidades y competencias para ser o saber participar en sociedad, es decir, formar para que los estudiantes alcancen una madurez humana que, de acu erdo con Luque (2019), en Ia formación de I a ci udadanía -tarea de t oda la vida- se requiere ejercer no so lo inteligencia para co nocer, sino t ambién v oluntad par a poner los en 
práctica. Se trata por tanto, no so lo de informar sino más bien de educar un ca rácter cívico. Como diría el humorista g ráfico e historietista argentino Quino y creador de Mafalda, "Educar es más difícil que enseñar, porque para enseñar usted necesita saber, pero para educar se precisa ser". En c uestiones relativas a la participación ciudadana y I a co nvivencia dem ocrática es necesario, $m$ ás aún en estos tiempos, tener conocimientos y saber buscar información pero, sobre todo, saber ser.

\section{Referencias}

Arnstein, S . R . (1969). A I adder of c itizen par ticipation. Journal of the American Institute of Planners, 35(4), 216-224. https://doi.org/10.1080/01944366908977225

Bakker, T. P. y De Vreese, C. H. (2011). Good news for the future? Young people, Internet use, and po litical $p$ articipation. Communication research, 38(4), 45 1-470. https://doi.org/10.1177/0093650210381738

Baño, R. (1998). Participación ciudadana: Elementos conceptuales. En Enrique Correa y Marcel Noé (Editores) Nociones De Una Ciudadanía Que Crece (pp 15-37). LOM.

Bennett, W. L., Wells, C. y Freelon, D. (2011). Communicating c ivic eng agement: C ontrasting models of citizenship in the youth web sphere. Journal of communication, 61(5), 835-856. https://doi.org/10.1111/j.1460-2466.2011.01588.x

Buitrago, É J. C., Yepes, O. C. R. y Matamoros, L. V. G. (2007). Historia, concepto y estructura de los derechos económicos, sociales y culturales. Revista Estudios Socio-Jurídicos, 9 (Esp), 77-108.

Calzada, I . ( 2017). D o d igital s ocial $n$ etworks foster c ivilian par ticipation am ong $m$ illennials? kitchenware r evolution and $15 \mathrm{M}$ democratic regeneration cases. Glocalism: Journal of Culture, Politics and Innovation. Special Issue: 'Beyond Democracy: Innovation as Politics', (3) https://doi.org/10.12893/gicpi.2017.3.4

Cohen, E . F . y Ghosh, C . ( 2019). Citizenship. C ambridge, U K; Me dford, M A: P olity. http://bvbr.bibbvb.de:8991/F?func=service\&doc_library=BVB01\&local_base=BVB01\&doc _number $=031306497 \&$ sequence $=000001 \&$ line_number $=0001 \&$ func_code $=D B \_R E C O R D$ S'\&service_type=MEDIA

Costa, P. (2006). Ciudadanía. Marcial Pons.

Delgado-Algarra, E. J . (2015). C onocimiento g local y pensamiento c rítico e n la ed ucación del siglo XXI: I nternational J ournal of $\mathrm{E}$ ducational $\mathrm{R}$ esearch and Innovation. International Journal of Educational Research and Innovation (IJERI), 4, I-V

Delgado-Algarra, E. J., Eloy López-Meneses, E., Bernal-Bravo, C. y Vázquez-Cano, E, (2020a) Educación para la ciudadanía y el desarrollo de agentes sociales proactivos y críticos. En M. ${ }^{a}$ M. Fernández-Martínez, A. Luque de la Rosa, J. J. Carrión-Martínez, I. M. ${ }^{a}$ RománSánchez, (Coords.) Innovación docente en la universidad y realidades emergentes, (pp.25- 40). Octaedro 
Delgado-Algarra, E. J., Aguaded, I., Bernal-Bravo, C. y Lorca-Marín, A. A. (2020b). Citizenship and Pluriculturalism A pproaches of $\mathrm{T}$ eachers in the $\mathrm{H}$ ispanic an $\mathrm{d} \mathrm{J}$ apanese $\mathrm{C}$ ontexts: $\begin{array}{llllll}\text { Higher E ducation R } & \text { esearch. } & \text { Sustainability, } & 12 & \text { (8), } 1 & -20 .\end{array}$ https://doi.org/10.3390/su12083109

Delgado-Algarra, E. J., Bernal-Bravo, C. y López-Meneses, E. (2019a). Multicultural competence and $\mathrm{c}$ osmopolitan $\mathrm{c}$ itizenship in $\mathrm{t}$ he $\mathrm{H}$ ispanic-Japanese $\mathrm{c}$ ontext of $\mathrm{H}$ igher $\mathrm{E}$ ducation. NAER: Journal of New Approaches in Educational Research, 8(2), 1 66-183. https://doi.org/10.7821/naer.2019.7.425

Delgado-Algarra, E. J ., Román-Sánchez, I. M., Ordónez-Olmedo, E . y Lorca-Marín, A. A . (2019b). International MOOC Trends in Citizenship, Participation and Sustainability: Analysis of T echnical, D idactic a nd C ontent D imensions. Sustainability, 11, 1 -19. https://doi.org/10.3390/su11205860

García-Galera, M. C., D el-Hoyo-Hurtado, M. y Fernández-Muñoz, C . (2014). J óvenes comprometidos en la Red: el papel de las redes sociales en la participación social activa. Comunicar, 22(43), 35-43. http://dx.doi.org/10.3916/C43-2014-03

González-Andrío Jiménez, R., Bernal Bravo, C. \& Palomero llardia, I. (2020 septiembre). Uso de las $r$ edes s ociales e ntre los j óvenes y c iudadanía digital: a nálisis t ras I a C OVID-19. Revista de Investigación en Didáctica de las Ciencias Sociales, 7. https://doi.org/10.17398/2531-0968.07.64

González, M .G., Becerra, M. T. y Berenice Yanez, M. (2016). Ciberactivismo: Nueva forma de participación para estudiantes universitarios. Comunicar, 24(44), 47-54. https://doi.org/10.3916/C46-2016-05

Heater, D. (2007). Ciudadanía : Una breve historia. Alianza.

Hernández Merayo, E. (2011). El c ompromiso c ívico y político de I os jóvenes y el rol de las nuevas t ecnologías en educación: Modelos de e -democracia. REDIE. Revista Interuniversitaria De Formación Del Profesorado, 25(2), 101-124. https://www.redalyc.org/articulo.oa?id=27422047006

Ichilov, O. (2013). Citizenship and citizenship education in a changing world Taylor and Francis. https://doi.org/10.4324/9781315822006

Jover, G . ( 2016). E ducación y c iudadanía: El c ompromiso c ívico de I os j óvenes es pañoles. Education in the Knowledge Society, 2(1) https://doaj.org/article/929e45d1065d405f92e49e7ec41f30da

La Porte, T. (2019). Las reglas del "nuevo poder de influencia":Un análisis de las estrategias de think tanks globales desde una perspectiva comunicativa. Austral Comunicación 8,(1), 936. https://doi.org/10.26422/aucom.2019.0801.lap

Lizcano Fernández, F. ( 2012). Conceptos de ciudadano, c iudadanía y c ivismo. Polis (32) http://journals.openedition.org/polis/6581

Luque, D. (2019). La traición a I a dem ocracia. Ensayo s obre las relaciones en tre uni versidad, democracia y ciudadanía desde la idea de t raición. Educatio Siglo XXI, 37(1 Mar-Jun), 21-40. https://doi.org/10.6018/educatio.362951

Marshall, T. H., \& Bottomore, T. (1992). Citizenship and social class. Alianza Editorial. 
Milbrath, L. W. (1965). Political participation: How and why do people get involved in politics? Chicago: Rand McNally.

Pagés, J. (2002). A prender a ens eñar historia y ciencias sociales: el currículo y la didáctica de las ciencias sociales. Pensamiento Educativo, Revista De Investigación Latinoamericana (PEL), 30 (I), 255-269. http://www.redae.uc.cl/index.php/pel/article/view/26411

Parés Franzi, M. (2009). Participación y calidad democrática: Evaluando las nuevas formas de democracia participativa. España: Ariel.

Prados, M. Á. H., Gómez, M. S., Ayala, E. S., \& Fuentes, J. L. (2015). Retos de la educación de nuestro s iglo: $\mathrm{E}$ ducación par a la vida, interculturalidad y $\mathrm{r}$ esponsabilidad tecnológica. Educación, Desarrollo y Cohesión Social, 150, 317 https://doi.org/5ebac5c1e4b04ec536ffdac2

Prensky, M. ( 2001). Digital nat ives, di gital i mmigrants. On the Horizon, 9(5). https://www.marcprensky.com/writing/Prensky\%20\%20Digital\%20Natives,\%20Digital\%20Immigrants\%20-\%20Part1.pdf

Ribble, M. S., B ailey, G. D ., \& Ross, T. W. (2004). D igital c itizenship: A ddressing ap propriate technology behavior. Learning \& Leading with Technology, 32(1). https://files.eric.ed.gov/fulltext/EJ695788.pdf

Robles Mor ales, J. M. (2009). Ciudadanía digital: Una introducción a un nuevo concepto de ciudadano (1.ed. en I engua $\mathrm{C}$ astellana. ed.). Editorial U OC. http://www.digitaliapublishing.com/a/19977/

Rowbottom, J. (2006). Me dia freedom and p olitical debate in the di gital era. The Modern Law Review, 69(4), 489-513.

Sabucedo, J. M. (1988). Participación política. Psicología política, 165-194.

Sádaba, I. ( 2012). A cción c olectiva y m ovimientos sociales e $\mathrm{n} I$ as $\mathrm{r}$ edes di gitales. as pectos históricos y m etodológicos. Arbor: Ciencia, Pensamiento Y Cultura, 188(756), 781-794. https://doi.org/10.3989/arbor.2012.756n4011

Touriñán López, J. M. (2001). Tecnología digital y sistema educativo: el reto de la globalización.Revista de Educación. (Número extraordinario), 217-230.

Vizcaíno-Laorga, R., C atalina-García, B ., \& de Ayala-López, M. L. (2019). Participación y compromiso de los jóvenes en el entorno digital. usos de las redes sociales y percepción de sus consecuencias. Revista Latina De Comunicación Social, (74), 554-572. https://doi.org/10.4185/RLCS-2019-1345

Westheimer, J., \& Kahne, J. (2004). What kind of citizen? the politics of educating for democracy. $\begin{array}{lllll}\text { American Educational Research Journal, 41(2), } 237 & -269 .\end{array}$ https://doi.org/10.3102/00028312041002237

\section{Referencias legislativas}

Constitución Española. B oletín O ficial del Estado núm. 311, de 29 de diciembre de 1978, pp. 29313 a 29424. https://www.boe.es/eli/es/c/1978/12/27/(1) 
Ley 39/2015, de 1 de octubre, del Procedimiento Administrativo Común de las Administraciones Públicas. Boletín O ficial del Estado núm. 236, de 2 de oc tubre de 2015, p p. 89343 a 89410. https://www.boe.es/eli/es///2015/10/01/39

Ley 50/1997, de 27 de noviembre, del Gobierno. Boletín Oficial del Estado núm. 285, de 28 de noviembre de 1997, pp. 35082 a 35088. https://www.boe.es/eli/es///1997/11/27/50

Orden PRE/1590/2016, d e 3 de octubre, p or I a q ue s e publica e I Acuerdo de I C onsejo de Ministros de 30 de septiembre de 2016, por el que se dictan instrucciones para habilitar la participación pública en el proceso de elaboración normativa a través de los portales web de I os dep artamentos ministeriales. B oletin O ficial de I E stado núm . 241, de 5 de octubre de 2016, pp 71075 a 71077 . https://www.boe.es/eli/es/o/2016/10/03/pre1590

Real Decreto 126/2014, de 28 de febrero, por el que se establece el currículo básico de Educación Primaria. B oletín O ficial d el Estado, n úm. 52, de 1 d e marzo de 2014, pp. 19349 a 19420. https://www.boe.es/eli/es/rd/2014/02/28/126

Real Decreto 1105/2014, de 26 de diciembre, por el que se establece el currículo básico de la Educación Secundaria Obligatoria y del Bachillerato. Boletín Oficial del Estado, núm. 3, de 3 de enero de 2015, pp.169 a 546. https://www.boe.es/eli/es/rd/2014/12/26/1105

\begin{tabular}{l} 
Publicado bajo licencia internacional Creative Commons Attribution-NonCommercial-ShareAlike4.0 \\
(CC-BY-NC-SA 4.0). Se permite copiar, usar, distribuir, transmitir y exhibir públicamente, \\
siempre que: i) se reconozca la a utoría y la fuente or iginal de publicación (revista, ed itorial y \\
URL del trabajo); ii) no se utilice con fines comerciales; iii) se mencione la existencia y \\
especificaciones de esta licencia. \\
\hline
\end{tabular} 